Article original

\title{
Biopsie du ganglion sentinelle après chimiothérapie néoadjuvante dans le cancer du sein
}

\author{
Sentinel lymph node biopsy after neoadjuvant chemotherapy in breast cancer
}

Khadidja Brahmi 1,2, M'hamed Mohamed Salaheddine Seddiki ${ }^{1,2}$, Merouane Boukrissa ${ }^{1,2,3}$, Bahia Merad-Tabet $^{4}$, Noureddine Bachir Bouiadjra 1,2

${ }^{1}$ Faculté De Médecine d'Oran, Université D'Oran.

${ }^{2}$ Clinique chirurgicale A, centre hospitalier et universitaire d'Oran.

${ }^{3}$ Service des urgences chirurgicales, centre hospitalier et universitaire d'Oran.

${ }^{4}$ Anatomopathologie, secteur privé, Oran.

\section{MOTS CLÉS}

Cancer du sein; ganglion sentinelle; curage axillaire; chimiothérapie néoadjuvante.

\section{Résumé}

Introduction - La technique du ganglion sentinelle dans le cancer du sein est appliquée aux lésions ne dépassant pas $50 \mathrm{~mm}$ (T1- T2), et controversée en présence d'adénopathie axillaire et après une chimiothérapie néo adjuvante. L'évaluation de cette technique se fait par un taux d'identification supérieur à 90\% et un taux de faux négatif entre 5 et 10\%. L'identification se fait par deux traceurs, un colorant et/ ou un radio-isotope. L'objectif est d'étudier la faisabilité de la biopsie du ganglion sentinelle après une chimiothérapie néo adjuvante dans les grosses tumeurs non inflammatoires ni métastatiques, avec et sans adénopathie axillaire.

Matériels et méthodes - Il s'agit d'une étude prospective menée à la clinique chirurgicale A du Centre Hospitalier Universitaire d'Oran entre janvier 2006 et avril 2009. Ont été incluses des patientes atteintes d'un carcinome mammaire infiltrant classé T2-T3 N0-N1 MO et traitées par une chimiothérapie néo adjuvante. Nous avons exclu les cancers récidivant, controlatéral et inflammatoire, une chirurgie antérieure, la grossesse, le cancer chez l'homme et une allergie au bleu patenté. Nous avons identifié le ganglion sentinelle par le bleu patenté. Un curage axillaire systématique et une mastectomie étaient réalisés. Les résultats étaient étudiés par rapport au statut ganglionnaire et à la réponse à la chimiothérapie.

Résultats - L'analyse a porté sur 71 patientes. La taille tumorale moyenne était de 4 $\mathrm{cm}$. Le taux d'identification était de $97.2 \%$ et le taux de faux négatifs de $8.6 \%$. Le taux d'identification était de $100 \%$ chez les patientes NO versus $95.3 \%$ chez les N1. Le taux de faux négatifs était élevé chez les $\mathrm{N} 1$, sans différence statistiquement significative $(p=0.11)$. En cas de bonne réponse à la chimiothérapie et de ganglions négatifs, le taux d'identification était élevé et le taux de faux négatifs bas.

'Auteur correspondant: brahmiskhadidja@yahoo.fr 
Conclusion - La biopsie du ganglion sentinelle serait possible après chimiothérapie néo adjuvante dans le cancer du sein invasif évolué localement, opérable, non inflammatoire ni métastatique.

\section{KEY WORDS \\ Breast cancer; sentinel lymph node; axillary dissection; neoadjuvant chemotherapy.}

\begin{abstract}
Introduction - Sentinel lymph node biopsy in breast infiltrating cancer is indicated for lesions not exceeding $50 \mathrm{~mm}$ (T1-T2) and controversial in the presence of axillary adenopathy and after neo adjuvant chemotherapy. Sentinel lymph node biopsy is assessed by an identification rate greater than $90 \%$ and a false negative rate between $5 \%$ and $10 \%$. A lymphophilic tracer, a patented blue dye and / or a radioisotope make the identification.
\end{abstract}

This study aims to investigate the feasibility of sentinel node biopsy after neo adjuvant chemotherapy in large non-inflammatory or metastatic tumors with and without axillary adenopathy.

Patients and methods - This prospective study was carried out in the surgical Clinic "A" of Oran University Hospital from January 2006 to April 2009. Patients with infiltrating breast carcinoma classified T2-T3 NO-N1 MO and treated with neo adjuvant chemotherapy were included. We have excluded recurrent or contralateral cancer, previous surgery, pregnancy, cancer in men and patent blue allergy.

The sentinel lymph node was identified by patent blue; mastectomy and axillary dissection were performed. The results were studied according to lymph node status and response to chemotherapy.

Results - Seventy-one patients participated in the study. The mean tumor size was 4 $\mathrm{cm}$. The identification rate was $97.2 \%$ and the false negative rate was $8.6 \%$. In patients N0, the identification rate was higher than the $\mathrm{N} 1$ (100\% vs $95.3 \%)$. The rate of false negatives was higher in the $\mathrm{N} 1$ group, with no statistically significant difference $(\mathrm{p}=$ 0.11). In the case of a good response to chemotherapy, the rate of identification was high and the rate of false negatives was low in patients with negative lymph nodes.

Conclusion - Sentinel lymph node biopsy is possible after neo adjuvant chemotherapy in locally advanced, operable, non-inflammatory and non-metastatic invasive breast cancer.

\section{Introduction}

L'envahissement ganglionnaire reste le principal facteur pronostique définissant le risque métastatique. Il a un rôle déterminant dans l'indication des traitements adjuvants [1]. L'exploration chirurgicale ganglionnaire axillaire systématique est indiquée en cas de carcinome mammaire infiltrant. Le curage axillaire (CA) est un geste chirurgical de référence pour obtenir cette information [2].Cependant, le CA est un geste chirurgical invasif grevé d'une morbidité non négligeable [3]. Il entraîne une morbidité à court terme, caractérisée essentiellement par des lymphocèles et des douleurs. La morbidité à long terme est caractérisée par une limitation des mouvements de l'épaule, une sensation de bras lourd et de moindre force, des douleurs et un lymphœdème du membre supérieur. Le taux de lymphocèles axillaires après curage axillaire est très variable, variant de 4 à $90 \%$ [4].
Afin de limiter la morbidité du geste axillaire en garantissant la sécurité carcinologique tout en obtenant une information pronostique, la technique du ganglion sentinelle (GS) a été développée. Le concept de GS, est apparu comme le premier relais théorique sur la voie de drainage lymphatique du sein $[5,6]$ et donc le premier relais théorique d'arrêt pour les cellules cancéreuses métastatiques. L'hypothèse est que si ce relais ganglionnaire sentinelle, identifié par l'injection de produit lymphotropes, ne contient pas de cellules cancéreuses, les autres ganglions de l'aisselle sont également indemnes et le curage peut être évité [2].Plusieurs études ont montré une morbidité significativement diminuée de la technique du GS comparée au CA [7].

Il existe deux types de méthode de détection, la méthode colorimétrique, utilisant le Bleu Patent qui est le triphénil méthane à 2,5\% et la méthode isotopique utilisant du technétium 99 métastable $(99 \mathrm{mTc})$ couplé à des colloïdes. Le GS peut donc être bleu 
et/ou radio détecté quand les deux techniques sont utilisées de manière combinée [8]. L'injection des produits se fait généralement en intra parenchymateux superficiel soit en péritumoral, soit en Péri aréolaire $[8,9,10]$. Des réactions allergiques, parfois graves, ont été décrites dans 1.1 à 2\% par le colorant bleu[11].

Un examen extemporané est réalisé en per opératoire qui a pour objectif de diagnostiquer une atteinte macro métastatique (>2mm) du GS, rendant possible le curage axillaire complémentaire dans le même temps opératoire [2]. Sa sensibilité pour la détection des macro métastases est de $63 \%$ [12]. Tous les GS doivent être examinés séparément à l'examen histologique standard pour confirmer la malignité ou rechercher une éventuelle métastase sur les GS négatifs à l'examen extemporané. L'immunohistochimie $(\mathrm{IHC})$ recherchera sur les GS négatifs une micro métastase $(0.2$ à $2 \mathrm{~mm}$ ) méconnue à l'histologie standard ou des cellules tumorales isolées $(<0.2 \mathrm{~mm})$ [13].

Les indications actuelles de la biopsie du GS pour les cancers invasifs concernent les lésions unifocales égales ou inférieures à 50 $\mathrm{mm}$, en absence d'adénopathie axillaire (cNO) ou si elle existe (cN1), une échographie et une cytologie ou biopsie ganglionnaire doit être négative [14, 15,16]. Cette technique reste controversée en cas d'adénopathie axillaire $\mathrm{cN} 1$, après une chimiothérapie néo adjuvante $(\mathrm{CN})$ et sur des tumeurs de plus de $50 \mathrm{~mm}$. Sa fiabilité se mesure sur le taux d'identification (TI) et le taux de faux-négatifs (TFN).

La CN représente une alternative thérapeutique pour une réduction tumorale afin de réaliser une chirurgie adéquate et traiter précocement la maladie micro métastatique [17].

L'objectif de notre étude était d'étudier la faisabilité de la biopsie du GS après chimiothérapie néo adjuvante, en cas de tumeur localement évoluée, opérable, non métastatique classée T2-T3, NO-N1, MO.

\section{Matériels et méthodes}

Il s'agit d'une étude prospective menée à la Clinique Chirurgicale A du Centre Hospitalier Universitaire d'Oran (CHUO) entre janvier 2006 et avril 2009. Des patientes atteintes d'un carcinome mammaire infiltrant classé CT2-T3 NO-N1 MO et traitées par une $\mathrm{CN}$ ont été incluses. Nous avons exclu le cancer récidivant et inflammatoire, une chirurgie antérieure mammaire et axillaire, la grossesse et une allergie au bleu patenté.

\section{Définitions :}

- Le taux d'identification a été défini par le nombre de patientes chez lesquelles un ou plusieurs ganglions ont été identifiés sur le nombre total de patientes injectées.

- Le faux négatif (FN) est la situation où le ganglion sentinelle est négatif alors que le curage axillaire est positif. Le taux de faux négatifs (TFN) a été défini par le nombre de patientes FN sur le nombre total de patientes ayant des ganglions envahis.

Lors de l'intervention, nous avons utilisé le Bleu Patenté qui a été injecté en péritumoral et péri aréolaire. Le creux axillaire était abordé par une courte incision cutanée et après une dissection les ganglions sentinelles colorés étaient repérés à la vue. Après leur exérèse, ces GS étaient adressés au pathologiste pour un examen extemporané afin de rechercher une métastase. Un CA systématique était réalisé après une mastectomie totale. L'analyse anatomopathologique des pièces opératoires a permis de confirmer le caractère positif ou négatif des GS, de préciser le nombre de ganglions envahis sur le nombre de ganglions prélevés dans le CA et de rechercher le résidu tumoral. D'autres éléments ont été également recueillis (le type histologique, la taille tumorale, le grade histopronostique et la présence d'emboles vasculaires péritumoraux). Ces éléments anatomopathologiques ont été renseignés dans un compte-rendu avec la définition du stade selon la classification post opératoire TNM [13].

La réponse à la $\mathrm{CN}$ a été évaluée par la clinique(yTNM), par l'étude anatomopathologique(yPTNM) et sur le résidu tumoral (mammaire et ganglionnaire) pour apprécier l'effet thérapeutique de la CN selon la classification de Sataloff. Cette analyse a été complétée par une étude immunohistochimique $(\mathrm{HC})$ à la recherche de micrométastases sur les ganglions négatifs, méconnues par l'examen anatomopathologique standard. L'indication d'un traitement médical adjuvant était faite selon les résultats définitifs. Un suivi a été préconisé avec surveillance post thérapeutique afin de déceler les récidives locales et à distance.

La réponse histologique après $\mathrm{CN}$ a été évaluée par deux classifications principales : la classification de Chevallier [18] se basant sur un score évaluant la toxicité chez des patientes en cas de tumeur inflammatoire (T4d) et la classification de Sataloff [19] qui concerne le cancer du sein localement avancé avec une réponse histologique :

\section{Sur la tumeur:}

TA: réponse histologique complète $(\mathrm{RHC})$ ou "presque complète" ;

TB: plus de $50 \%$ de régression;

TC: moinsde $50 \%$ de régression;

TD: pas d'effet.

Sur les ganglions :

NA : pas de maladie résiduelle ;

NB : pas d'atteinte axillaire ou d'effet thérapeutique ;

NC : ganglion(s) positif(s) avec effet thérapeutique ;

ND : ganglions métastatiques et pas d'effet thérapeutique.

\section{Analyse statistique}

Les données recueillies ont été codées, saisies et exploitées en utilisant le logiciel Epilnfo6. Une analyse descriptive a été réalisée avec le calcul des moyennes et des écarts type pour les variables quantitatives et des pourcentages pour les variables qualitatives.

\section{Consentement éclairé}

Le protocole de cette étude a été soumis aux patientes après une 
information sur la procédure.

Un consentement éclairé a été signé par les patientes en présence d'un membre de leur famille.

\section{Résultats}

\section{- Répartition des patientes :}

Trois patientes ont été exclues de l'étude. Parmi ces trois patientes, deux ont été perdues de vue et la troisième est décédée à la suite d'un infarctus myocardique.

Soixante et onze (71) patientes ont été incluses dans l'analyse. L'âge moyen était de 49.2 ans avec des extrêmes allant de 26 à 72 ans. La taille tumorale moyenne était de $4 \mathrm{~cm}$ [3-7]. La classification TNM des patientes avant et après chimiothérapie néo adjuvante est représentée dans le tableau 1.

\section{- Caractéristiques histopathologiques}

Le carcinome canalaire infiltrant (CCl)représentait 83.1\%. Le nombre moyen de ganglions examinés dans le CA était de 13 [9 à 23]. Le CA était négatif dans $64.8 \%$ (46/71).

Figure 1. Identification du GS

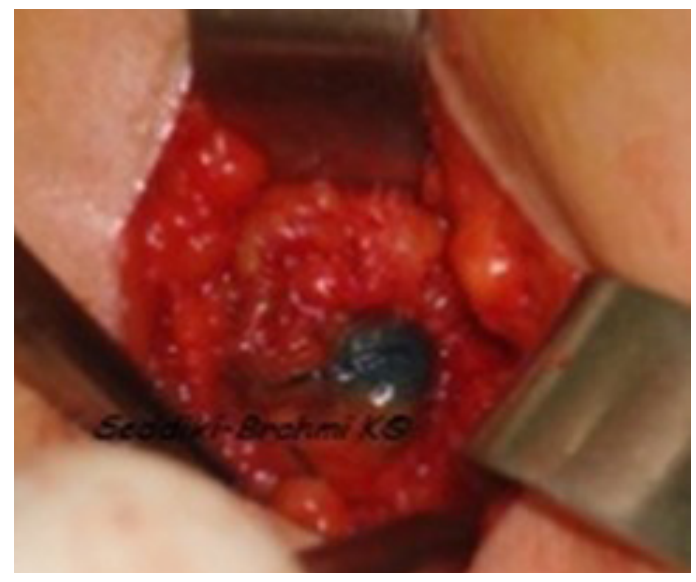

Figure 2. Identification du GS

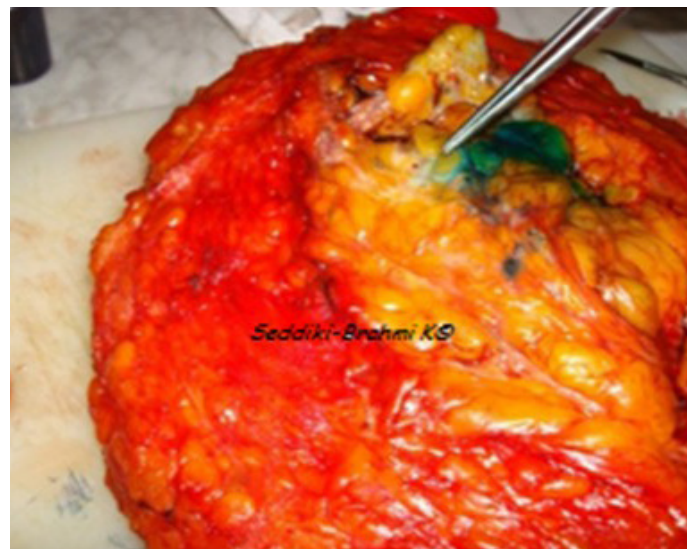

\section{- Biopsie du GS}

Le taux d'identification (TI) était de $97.2 \%$ (69/71) et le taux de faux négatifs de $8.6 \%(2 / 23)$. La valeur prédictive négative (VPN) était de $95.8 \%$ (46/48). Les deux échecs (2.8\%) d'identification du GS correspondaient à des patientes obèses avec un BMl égal à 29 et 30. Le nombre moyen de GS prélevés était de 1 [1 à 4] (Figure 1, Tableau 2).

L'examen extemporané per opératoire du GS avait une sensibilité de $94.1 \%$ et une spécificité de $88.5 \%$.

- Résultats du GS par rapport au statut ganglionnaire clinique initial Le TI était supérieur chez les patientes cNO par rapport aux pa-

Tableau 2 : Analyse des facteurs de risque de cancer du sein

\begin{tabular}{|l|c|c|c|}
\hline & OR & IC à 95\% & $p$ \\
\hline Age $<40$ ans & 0,99 & $0,62-1,55$ & NS \\
\hline Age $\geq 70$ ans & 0,95 & $0,53-1,71$ & NS \\
\hline Ménarchie précoce & 3,76 & $1,55-1,41$ & $<0,001$ \\
\hline Ménopause tardive & 0,12 & $0,05-0,24$ & $<0,001$ \\
\hline Nulliparité & 5,67 & $2,30-14,52$ & $<0,001$ \\
\hline Parité $>5$ & 0,31 & $0,21-0,47$ & $<0,001$ \\
\hline $\begin{array}{l}\text { Age tardif de la pre- } \\
\text { mière grossesse }\end{array}$ & 2,55 & $0,40-20,25$ & NS \\
\hline $\begin{array}{l}\text { Prise de contracep- } \\
\text { tifs oraux }\end{array}$ & 0,26 & $0,18-0,41$ & $<0,001$ \\
\hline $\begin{array}{l}\text { Allaitement ma- } \\
\text { ternel }\end{array}$ & 0,19 & $0,11-0,33$ & $<0,001$ \\
\hline Obésité $\left(\geq 30 \mathrm{~kg} / \mathrm{m}^{2}\right)$ & 2,76 & $1,63-4,71$ & $<0,001$ \\
\hline
\end{tabular}

tientes cN1 (100\% vs 95,3\%), sans différence statistiquement significative. Le TFN était plus élevé chez les patientes $\mathrm{CN} 1$ mais la différence entre les deux groupes n'était pas statistiquement significative $(\mathrm{p}=0.11)$.

\section{- Résultats du GS par rapport à la réponse clinique de la CN}

Le Tl était de 100\% (20/20) et le TFN de $9 \%$ chez les patientes ayant peu ou pas répondu à la $\mathrm{CN}$. Le Tl était de $94.7 \%$ (18/19)chez les patientes ayant une réponse clinique complète ou presque complète, et de $96.8 \%$ (31/32) chez les patientes ayant une réponse clinique de plus de $50 \%$ ( $\mathrm{p}=0.1$ ) ; le TFN était de $0 \%$ dans ces deux groupes.

- Résultats du GS par rapport à l'étude histologique (classification yPTNM).

Le TFN était meilleur chez les patientes n'ayant pas de métastases ganglionnaires (ypNO) ou ne dépassant pas trois ganglions métastatiques (ypN1) $(p=0.11)$. Le TFN augmentait dès qu'il y avait plus de 3 ganglions métastatiques. 
- Résultats du GS par rapport à l'évaluation du résidu tumoral (classification de Sataloff)

L'effet thérapeutique s'est présenté sous forme d'altérations microscopiques (fibrose, nécrose, foyers de calcifications : Figures 2 et 3).

Sur la tumeur : Le TI du GS était de 100\% chez les patientes qui avaient peu ou pas répondu à la chimiothérapie (TC-TD), par rapport aux patientes ayant eu une bonne réponse (TA- TB) $(\mathrm{p}=0.1)$. Inversement, Le TFN était de $0 \%$ en cas de bonne réponse (TA-TB) et de $13.3 \%$ en cas de mauvaise réponse (TC-TD), avec une différence statistiquement significative $(p=0.01)$.

Sur les ganglions : les patientes sans métastase ganglionnaire (NANB) ont un TI de 100\% par rapport aux patientes ayant une métastase ganglionnaire (NC- ND) $(p=0.3)$. Le TFN du GS était de $0 \%$ chez les patientes NA-NB et de $12.5 \%$ chez les patientes ND, avec une différence statistiquement significative $(\mathrm{p}=0.01)$.

Parmi les patientes qui avaient des GS négatifs, l'analyse en IHC avait retrouvé $21 \%$ de micro métastases et $17 \%$ de cellules isolées. Après un recul de 48 mois, les récidives axillaires

(RA) chez l'ensemble des patientes étaient de 5.7\% (4/69).

\section{Discussion}

La biopsie du ganglion sentinelle est actuellement le standard du traitement de l'aisselle pour les cancers invasifs du sein n'excédant pas les $50 \mathrm{~mm}[14,15,16]$. Dans la présente étude, le taux d'identification des GS était de $97.2 \%$ (69/71) et le taux de faux négatifs de $8.6 \%$ (2/23). Ces résultats concordent avec ceux de plusieurs études. En effet, dans une méta-analyse regroupant 21 études (1273 patientes) dans laquelle le GS avait été prélevé après une chimiothérapie néo adjuvante associé à un CA systématique, le taux de détection était de $91 \%$ avec un TFN de 12\% [20]. Ce TFN était plus faible (7.3\%) dans une autre méta-analyse regroupant 8059 patientes (69 études) ayant eu un GS suivi de CA systématique sans chimiothérapie préalable [21]. Dans l'étude GANEA portant sur 195 patientes présentant des tumeurs uniques traitées par chimiothérapie première et bénéficiant ensuite d'une chirurgie conservatrice mammaire avec un GS suivi d'un CA systématique, le TI du GS était de $90 \%$ et le TFN de $11.5 \%$ [22]. D'autres études ont rapporté un TFN $>10 \%$ et suggèrent de prélever au moins trois ganglions sentinelles [23].

Nos 2 échecs d'identification sont des patientes avec un BMI de 29 et 30 et les $\mathrm{FN}$ sont des patientes n'ayant pas répondu à la CN. La diffusion du bleu est souvent retardée et la zone de dissection difficile. Un envahissement massif du GS constitue un obstacle au drainage lymphatique [24].

Concernant le statut ganglionnaire clinique $(\mathrm{cN})$, les études rapportent un TFN du GS en cas de cNO- cN1, de $0 \%$ vs $9 \%$ [25], de $9 \%$ vs $15 \%$ [24] et de $0 \%$ vs $25 \%$ [26]. Nos résultats étaient comparables.

En tenant compte de la réponse à la CN, le TI du GS est plus élevé chez les patientes TC-TD et les patientes ayant une stérilisation des ganglions après l'effet thérapeutique de la chimiothérapie (NB) $[26,27]$ ce qui correspond à nos résultats. Ceci suggère que le statut ganglionnaire ne semble pas influencer le $\mathrm{TI}$ en cas de $\mathrm{CN}$.
Le TFN est inférieur chez les patientes TA-TB par rapport aux patientes TC- TD, $11.1 \%$ vs $12.3 \%$ [28].Le TFN est de $0 \%$ chez les patientes sans métastases ganglionnaires (NA- NB) et de $11.5 \%$ chez les patientes ayant des métastases ganglionnaires (NC- ND) [24].

Après chimiothérapie, des études ont montré que la $\mathrm{CN}$ est associée à un taux de $60 \%$ de CA négatif versus $39 \%$ en cas de chirurgie première $(\mathrm{p}<0.001)[29]$. Dans notre étude le CAétait négatif dans $64,8 \%(46 / 71)$.

Le faux négatif correspond à un éventuel risque de récidives axillaires (RRA) mais ce risque dépend d'autres facteurs pronostiques et prédictifs décisionnels : l'envahissement ganglionnaire, le grade tumoral, les récepteurs hormonaux, les emboles vasculaires, la surexpression de la protéine Her2 et l'âge < 35 ans [16].

Concernant les récidives axillaires (RA), lorsque le GS est négatif, le RRA est de $0.5 \%$ pour Krag [18] et de $0.4 \%$ pour Houvenaeghel [30]. La RA à 5 ans est de $0.5 \%$ en cas de GS micrométastatique et de $0.9 \%$ si le GS est macrométastatique [31]. Le grade histologique, les récepteurs hormonaux et l'âge étaient associés au risque de récidive locorégionale, de façon identique dans les 2 bras.

Dans l'étude ACOSOG Z0011 portant sur 891 patientes classées T1-T2, N0 clinique deux groupes ont été constitués en cas de GS métastatique : curage axillaire complémentaire versus abstention, une chirurgie conservatrice avec radiothérapie postopératoire et traitement adjuvant systémique obligatoire.La survie globale et la survie sans récidive à 6.3 ans étaient comparables dans les 2 groupes. Il n'y avait pas plus de RA dans le groupe abstention que dans le groupe curage axillaire complémentaire $(0.5$ versus $0.9 \%$ à 6.3 ans). La survie globale était de $91.8 \%(95 \% \mathrm{Cl}, 89.1 \%-94.5 \%)$ avec curage et de $92.5 \%$ (95\% Cl, 90.0\%-95.1\%) avec GS seul [31]. Ceci a été expliqué par le traitement adjuvant et la radiothérapie du sein. Après un recul de 9.3 ans, ces auteurs ont eu un taux de RA de $1.5 \%$ après GS seul, contre $0.5 \%$ après CA complémentaire (sans différence statistiquement significative). La survie globale et la survie sans récidive n'étaient pas statistiquement différentes dans les 2 groupes [32].

Dans une autre méta-analyse, réalisée sur 26870 patientes avec un GS métastatique seul et 103705 patientes avec un GS métastatique associé à un CA complémentaire, les auteurs n'ont pas retrouvé de différence en survie globale et sans récidive concernant le taux de RA [33].

Les sociétés savantes ne recommandent plus de CA complémentaire si le GS est métastatique (avec moins de 3 GS envahis) lorsqu'un traitement médical est prescrit en cas de chirurgie conservatrice complétée par une radiothérapie [34].

En ce qui concerne la prise en charge des micrométastases, dans l'essai IBCSG23-01 [35] pour GS micro métastatique, comportant 2 groupes de patientes atteintes d'un cancer classé T1-T2, N0 (92\% $<3 \mathrm{~cm}$ ), 464 patientes ont eu un GS avec CA versus $467 \mathrm{GS}$ seul. Un traitement adjuvant systémique était réalisé dans $99 \%$ des cas. Après un suivi médian de 5 ans, la survie sans récidive était de $87.8 \%$ dans le groupe GS seul versus $84.4 \%$ dans le groupe GS plus CA.

Le CA n'est pas recommandé pour les femmes atteintes d'un cancer du sein précoce, qui ont un ou deux GS métastatiques avec une 
chirurgie conservatrice du sein suivie d'une radiothérapie conventionnelle [36].

\section{Les limites de l'étude}

La mastectomie a été réalisée chez l'ensemble des patientes à cause de l'indisponibilité de la radiothérapie dans les délais dans un traitement conservateur. L'absence de repérage radiologique de la tumeur en cas de disparition tumorale sous chimiothérapie néo adjuvante était le deuxième motif.

Concernant les patientes ayant un ganglion axillaire cliniquement (cN1), nous n'avons pas réalisé une appréciation histologique. Actuellement, les référentiels recommandent une appréciation échographique et cytologique voire histologique [15].

\section{Conclusion}

La technique du ganglion sentinelle constitue désormais une avancée majeure dans la prise en charge du cancer du sein. Après une chimiothérapie néo adjuvante, elle pourrait être indiquée chez les patientes ayant une tumeur dépassant les $5 \mathrm{~cm}$ avec une adénopathie axillaire prouvée négative en histologie. Il semblerait à travers notre étude que plus de $50 \%$ des patientes ayant eu une chimiothérapie pourraient bénéficier de la biopsie du GS. Cette technique mini invasive peut réduire la durée d'intervention et d'hospitalisation, améliorer la qualité de vie par une diminution de la douleur post opératoire et une récupération rapide de la mobilité du membre supérieur. Elle peut également s'inscrire dans une désescalade chirurgicale dans la perspective d'augmenter les chances de conservation mammaire après une chimiothérapie néo adjuvante.

\section{Conflits d'intérêts}

Les auteurs déclarent n'avoir aucun lien d'intérêts.

\section{Références bibliographiques}

[1] Goldhirsch A., Wood W.C., Gelber R.D., and al. Meeting highlights: updated international expert consensus on the primary therapy of early breast cancer J Clin Oncol 2003; 21: 3357-3365.

[2] J.M. Classe, L. Sentilhes, I. Jaffré, M. Mezzadri, C. Lefebvre-Lacoeuille, M. Dejode, L. Catala, V. Bordes, F. Dravet, P. Descamps. Chirurgie des cancers invasifs du sein. Gynécologie Obstétrique et Biologie de la Reproduction 2010. V 39, 8S, 43-62.

[3] E. Barranger, M. Dejode, Y. Delpech. Place du curage axillaire complémentaire en cas de ganglion sentinelle métastatique. La Lettre du Sénologue $\bullet \mathrm{N}^{\circ} 75$ - janvier-février-mars 2017.

[4] Pogson C.J., Adwani A., Ebbs S.R. Seroma following breast cancer surgery Eur J Surg Oncol $2003 ; 23: 711-717$

[5] Krag D.N., and al. Surgical resection and radio localization of the sentinel lymph node in breast cancer using a gamma probe Surg Oncol 1993; $2: 335-340$

[6] Giuliano A.E., and al. Lymphatic mapping and sentinel lymphadenectomy for breast cancer Ann Surg 1994 ; 220 : 391-401.

[7] Houvenaeghel G. Impact des techniques chirurgicales sur le contrôle loco-régional et la survie. Cancer du sein. Compte-rendu du cours supé- rieur francophone de cancérologie Saint-Paul-de-Vence, janvier 2009. p. 239-62.

[8] J.-M. Classe, G. Houvenaeghel, C. Sagan. La détection du ganglion axillaire sentinelle appliquée au traitement du cancer du sein : le point en 2007. Journal de Gynécologie Obstétrique et Biologie de la Reproduction. Vol 36-N 4-Juin 2007p.329-337).

[9] Salmon RJ, Nos C, Lojodice F, Languille O. Ganglion sentinelle et cancer opérable du sein : utilisation du bleu patent. Étude pilote. Ann Chir 2000 ;125:253-8.

[10] Garcia-Manero M, Olartecoechea B, Royo P. Different injection sites of radionuclide for sentinel lymph node detection in breast cancer: single institution experience. Eur J Obst et Gynecol Reprod Biol 2010;153:185-7.

[11] Lyew MA, Gamblin TC, Ayoub M. Systemic anaphylaxis with intramammary isosulphan blue injection used for sentinel node detection under general asnesthesia. Anesthesiology 2000;93:1145-6.

[12] Tew K., and al. Meta-analysis of sentinel node imprint cytology in breast cancer Br J Surg $2005 ; 92$ : 1068-1080.

[13] Classification TNM des cancers du sein. AJCC Cancer Staging Manual. 7th ed. New York: Springer; 2010.

[14] Référentiel de l'AP-HP- Cancers du sein- Août 2014.

[15] Référentiels de l'AP-HP- Cancers du sein- Mars 2016.

[16] Cottu P (Institut Curie), Delaloge S. (Gustave Roussy). REFERENCIELS « Cancers et pathologies du sein ». Attitudes diagnostiques et thérapeutiques, protocoles de traitement 2016- 2017.

[17] Kaufmann M, HortobagyiGN, Goldhirsch A, et al. Recommandations from an international expert panel on the use of neoadjuvant systemic treatment of operable breast cancer: an update. J Clin Oncol 2006 : 1940-1949.

[18] Chevallier B, Roche H, Olivier JP, Chollet P, Hurteloup P. Inflammatory breast cancer. Pilot study of intensive induction chemotherapy (FEC-HD) results in a high histologic response rate. Am J Clin Oncol 1993;16(3):223-8.

[19] Sataloff DM, Mason BA, Prestipino AJ, Seinige UL, Lieber CP, Baloch Z. Pathologic response to induction chemotherapy in locally advanced carcinoma of the breast: a determinant of outcome. J Am Coll Surg 1995; 180(3):297-304.

[20] Xing Y., and al. Meta-analysis of sentinel lymph node biopsy after preoperative chemotherapy in patients with breast cancer $\mathrm{Br} J$ Surg 2006; 93 : 539-546.

[21] Kim T., Giuliano A.E., Lyman G.H. Lymphatic mapping and sentinel lymph node biopsy in early-stage breast carcinoma: a meta analysis Cancer $2006 ; 106: 4-16$

[22] Classe J.M., and al. Sentinel lymph node biopsy after neoadjuvant chemotherapy for advanced breast cancer: results of sentinel lymph node biopsy and neoadjuvant chemotherapy, a French prospective multicentric study J Clin Oncol 2009 ; 27 : 726-732.

[23] Kuehn T, Bauerfeind I, Fehm T et al. Sentinel-lymph-node biopsy in patients with breast cancer before and after neoadjuvant chemotherapy (SENTINA): a prospective, multicentre cohort study. Lancet Oncol 2013;14(7):609-18.

[24] Bordes V, Giard S, Mignotte H, RodierJF, Laveque J, Ferron G, Marchal F, Alran S, Ladonne JM, Cuisenier J, Dupre PF, Body G, Classe JM. Sentinel node biopsy after neoadjuvant chemotherapy in breast cancer. Bull Can. Volume 98, Number 5, 506-10, Décembre 2006. 
[25] Lang JE, Esserman IJ, Ewing CA, et al. Accuracy of selective sentinel lymphadenectomy after néoadjuvant chemotherapy: effect of clinical node status at presentation. J Am Coll Surg 2004; 199:856-62.

[26] Le Bouëdec G, Geissler B, Gimbergues P, Cachin F, Penault-Llorca F, Kwiatkowski F, Dauplat J, Maublant J. Sentinel lymph node biopsy for breast cancer after neoadjuvant chemotherapy : influence of nodal status before treatment. Bull Can. Volume 93, Number 4, 415-9, Avril 2006.

[27] Kinoshita T, Takasugi M, Iwamoto E, Akashi-Tanaka S. Sentinel lymph node biopsy examination for breast cancer patients with clinically negative axillary lymph nodes after néoadjuvante chemotherapy. Am J Surg 2006; 191: 225-9.

[28] Mamounas EP, Brown A, Anderson S, et al. Sentinel node biopsy after neoadjuvant chemotherapy in breast cancer: results from National Surgical Adjuvant Breast and Bowel Project Protocol B-27. J Clin Oncol 2005; 23: 2694-702.

[29] Gianni L, Baselga J, Eiermann W et al. Phase III trial evaluating the addition of paclitaxel to doxorubicin followed by cyclophosphamide, methotrexate, and fluo- rouracil, as adjuvant or primary systemic therapy: European Cooperative Trial in Operable Breast Cancer. J Clin Oncol 2009;27(15):2474-81.

[30] Houvenaeghel $G$ et al. Impact of completion axillary lymph node dissection in patients with breast cancer and isolated tumour cells or micro metastases in sentinel nodes. Eur J Cancer 2016). Volume 67, Pages 106-118.
[31] Giuliano A, Hunt KK, Ballman KV et al. Axillary dissection vs no axillary dissection in women with invasive breast cancer and sentinel node metastasis: a randomized clinical trial. JAMA 2011;305(6):569-75.

[32] Giuliano AE, Ballman K, McCall L et al. Locoregional recurrence after sentinel lymph node dissection with or withoutaxillary dissection in patients with sentinel lymph node metastases: long-term follow-up from the American College of Surgeons Oncology group (Alliance) ACOSOG Z0011 randomized trial. Ann Surg 2016;264(3):413-20.

[33] Li CZ, Zhang P, Li RW et al. Axillary lymph node dissection versus sentinel lymph node biopsy alone for early breast cancer with sentinel node metastasis: a meta-analysis. Eur J Surg Oncol 2015;41(8):958 66.

[34] Goldhirsch A, Wood WC, Coates AS, Gelber RD, Thürlimann B, Senn $\mathrm{HJ}$; Panel members. Strategies for subtypes--dealing with the diversity of breast cancer: highlights of the St. Gallen International Expert Consensus on the Primary Therapy of Early Breast Cancer 2011. Ann Oncol 2011;22:1736-47.

[35] Galimberti V1 and al. Axillary dissection versus no axillary dissection in patients with sentinel-node micro metastases (IBCSG 23-01): a phase 3 randomised controlled trial. Lancet Oncol. 2013 Apr;14(4):297305. doi: 10.1016/S1470-2045(13)70035-4. Epub 2013 Mar 11.

[36] Lyman GH1 and al. Sentinel lymph node biopsy for patients with early-stage breast cancer: American Society of Clinical Oncology clinical practice guideline update. J Clin Oncol. 2014 May 1;32(13):1365-83. doi: 10.1200/JCO.2013.54.1177. Epub 2014 Mar 24. 
